KAR definitiv identisch sind?) Ist eine "Anforderung" überhaupt sinnvoll beschreibbar, ohne die Person zu betrachten, an die sich die Anforderung richtet? (Stellt z. B. die Aufgabe, mit geschlossenen Augen geradeaus zu gehen, für Leistungsturnerinnen und KleinhirnSchlaganfall-Patienten nicht extrem unterschiedliche Gleichgewichtsanforderungen?) Wie geht NEUMAIER mit solchen Fragen um? Er spricht sie an und diskutiert sie in der Regel, soweit es als notwendig erscheint; er lässt sie allerdings immer dann als offene Fragen stehen, wenn die Motorikforschung (noch) keine griffigen Antworten anzu- bieten hat - und dies ist wohl die seriöse Form der Darstellung eines Problemfeldes, zu dem der bestehende Forschungsbedarf unzweifelhaft erheblich ist.

Zusammengefasst steht zu hoffen, dass die von NEumaier vorgeschlagene Systematik ihren Weg in die Praxis des Koordinationstrainings finden wird. Der von NeUMAIER/MECHLING geplanten Buchreihe ist daher zu wünschen, dass die sportartspezifischen Nachfolgebände eine vergleichbare Qualität erreichen werden wie der hier besprochene erste Band.

ERNST-JOACHIM HOSSNER

\title{
Die Sportwissenschaft im Internet
}

\section{Histe der abgeschlossenen Dissertationen/Habilitationen und der Literatur-Neuerscheinungen}

Die bislang in der Regel einmal im Jahr in der Sportwissenschaft publizierten Listen der sportwissenschaftlichen Dissertationen und Habilitationen sowie der Literatur-Neuerscheinungen lassen sich seit Beginn dieses Jahres über die Homepage des Bundesinstituts für Sportwissenschaft unter der Adresse www.bisp.de abrufen. Sie befinden sich unter dem Button "Aktuelles", Unterrubrik "Fachinformation".

\section{濇 Inhaltsübersicht und Kurzfassungen der Beiträge}

Über die Homepage des Hofmann-Verlags lassen sich Kurzfassungen der Hauptbeiträge in deutsch, englisch und französisch, sowie die Inhaltsverzeichnisse des aktuellen Jahrgangs der Sportwissenschaft unter der Adresse wwwhofmann-verlag.de in der Rubrik "Sport”, im Unterverzeichnis "Zeitschriften" abrufen. 